\title{
DECISIONES DE COMPRA Y CONSUMO DE BEBIDAS LIGHT NO ALCOHÓLICAS EN EL DISTRITO METROPOLITANO DE QUITO
}

\author{
DECISIONS OF PURCHASE AND CONSUMPTION OF LIGHT NON-ALCOHOLIC \\ BEVERAGES IN THE METROPOLITAN DISTRICT OF QUITO
}

\author{
Alcívar Pila Tituaña \\ Universidad Tecnológica Indoamérica \\ alcibarpila@uti.edu.ec \\ Irina Freire Muñoz \\ Universidad Tecnológica Indoamérica \\ irinafreire@uti.edu.ec
}

RECIBIDO: 03 / 04 / 2017

APROBADO: 19 / 05 / 2017

\begin{abstract}
Resumen
Actualmente existe una problemática evidenciada en el aumento de la mortalidad a causa de enfermedades no transmisibles provocadas por el sobrepeso y la obesidad, por lo que la idea del consumidor de llevar una vida más saludable con alimentación baja en calorías, grasa y azúcares, ha llevado a una respuesta masiva de la industria alimenticia que ofrece productos especialmente diseñados para responder esas expectativas y necesidades concretas de salud y bienestar. El presente estudio se orienta a esclarecer cómo las decisiones de compra y consumo de bebidas light no alcohólicas están relacionadas con el estilo de vida light y la cultura del sentirse y verse bien y cómo se manifiestan estas decisiones en los consumidores del D.M de Quito. Entre los resultados del estudio se pudo evidenciar que las principales motivaciones que inciden en la decisión de compra y consumo de bebidas light no alcohólicas es la salud y el bienestar físico y estético, aunque desconociendo sobre los verdaderos beneficios o perjuicios de estos productos. La investigación utiliza la técnica de la encuesta y corresponde a un enfoque cuantitativo con un diseño no experimental, temporalidad transeccional y alcance descriptivo y correlacional.
\end{abstract}

\section{Palabras clave}

Estilo de vida, salud, bienestar, perjuicio, producto.

\begin{abstract}
There's a problem evidenced in the current increase in mortality due to non-communicable diseases caused by overweight and obesity, that lead the consumer to search for healthier way of life, with low calorie, fat and sugar free diet that has created a massive response from the food industry that offers to the market products specially designed to meet the expectations and specific health and wellness needs of the consumer. The present study aims to clarify how the decisions of purchase and consumption of nonalcoholic light drinks are related to the light lifestyle and the culture of feeling and looking good and how these decisions are manifested in consumers of the city of Quito. Among the results of the study it was possible to demonstrate that the main motivations that influence the purchase and consumption of nonalcoholic light drinks are health and physical and aesthetic wellbeing, although the consumer do not know about the true benefits or damages of these products. This study uses the survey as research technique and corresponds to a quantitative approach with a non-experimental design, transectional temporality and descriptive and correlational scope.
\end{abstract}




\section{Keywords}

Lifestyle, health, welfare, detriment, product.

\section{Introducción \\ Del ciudadano de la salud al ciudadano light}

En la actualidad existe una problemática evidenciada en el aumento de la mortalidad a causa de enfermedades no transmisibles provocadas por el sobrepeso y la obesidad. Varias investigaciones realizadas por la Organización Mundial de la Salud en cooperación con universidades médicas norteamericanas pronosticaron que a partir del año 2010 se superarán las 180.000 muertes al año en el mundo a causa de daño cardiovascular, hipertensión arterial, lesiones óseas, cardiopatías, entre otras patologías provocadas por la alimentación procesada con concentraciones altas de azúcar y grasa. (V. Malik, B. Popkin, G. Bray, J. Després, F. Hu, 2010) Ante este contexto, surge la preocupación por mantener una vida más saludable y enfocada en el consumo de alimentos bajos en grasa, azúcares no procesados y bajos en sodio, apareciendo así la alimentación light o ligera.

El Laboratorio Nacional de Protección a los consumidores PROFECO (México) se ha dedicado durante varios años a estudiar la calidad de los productos de consumo generalizado y de impacto en la nutrición, seguridad y economía de las personas. Uno de sus estudios en el 2008 estuvo encaminado específicamente a los productos denominados light, o "ligeros", y da cuenta que el origen de estos productos se da en 1897, cuando el químico ruso Constantin Fahlberg descubre la sacarina, una substancia 300 veces más dulce que el azúcar, lo que causaría una revolución en la industria alimenticia de los próximos dos siglos. Esto llevaría al inicio de lo que hoy se conoce como productos alimenticios light. (Sánchez, 2008).

En el siglo XX, en Estados Unidos y parte de Europa se dio un interés creciente de las personas por mantenerse saludables, lo que provocó que las empresas alimenticias necesitaran modificar la composición de los alimentos procesados y esforzarse por alcanzar el desarrollo tecnológico y productivo que el mercado exigía. De esta manera no solo hubo un cambio en la utilización de la sacarina, en lugar del azúcar, sino en sustituir las grasas por otras artificiales que aporten menos calorías que la grasa normal. Según Sánchez (2008), ya en los años 1980 aparecen los alimentos light más representativos, principalmente en bebidas refrescantes sin azúcar.

Paralelamente a los estudios científicos, la investigación social referente a la cultura de la modernidad, sostiene que llevar una vida más saludable no es exclusivamente un pensamiento orientado a evitar enfermedades, sino a la necesidad de adquirir un cuerpo física y socialmente aceptado, es decir, en la sociedad del consumo actual existe una tendencia del sujeto consumidor por el cuidado personal en la dualidad verse/sentirse bien. (Arízaga, 2003).

En estad dinámica aparece el estilo de vida light, en el cual el comportamiento del consumidor está relacionado con el sentimiento de bienestar mental, psicológico y social, más allá del bienestar exclusivamente físico. (Bauman, 2003).

Para Janet Rico (2011) el "estar en forma" también ha promovido esta nueva cultura particularmente denominada como light o ligera, atendidas por las empresas dedicadas a la mercadotecnia y la publicidad, enfocadas principalmente a remarcar su interés por la salud y la importancia que representan los productos ofertados como saludables para los millones de consumidores en el mundo. Según M. Max-Neef (1998), podría interpretarse a estos productos como satisfactores que cubren una necesidad, y que corresponde a un sistema que opera en función del momento histórico - cultural en el que el individuo se desarrolla, por lo que se produce un proceso de simultaneidades, complementariedades y compensaciones como características de la dinámica de satisfacción de necesidades (Boltvinik 2014) . 


\section{Bebidas light no alcohólicas: ¿saludable o no saludable?}

En efecto, la idea del consumidor de llevar una vida más saludable con alimentación ligera, baja en calorías de grasa y azúcares, ha llevado a una respuesta masiva de la industria alimenticia que ofrece al mercado productos especialmente diseñados para responder esas expectativas y necesidades concretas de salud y bienestar, pero según S. Hall, a partir de 1990 la cultura de consumo se enfocó en la elección y diferenciación de productos según su estilo de vida y no por clase social de pertenencia. (Arízaga, 2003)

Pues bien, se denomina light a todos aquellos alimentos sólidos o líquidos que, además de presentar una reducción de carbohidratos y azúcares totales, del 25 - 30\%, y una reducción mayor de 5 gr. /100 gr. de producto, para los sólidos, y de 2,5 gr. /100 gr., para los líquidos, también debe presentar una reducción de grasas totales, mínimo del $25 \%$, y al menos el $30 \%$ de sodio del alimento sólido o líquido original. (Sánchez, 2008)

Las bebidas light no alcohólicas corresponden al tipo de producto alimenticio de consumo personal no duradero que no contiene alcohol, al cual se le ha realizado un proceso de reducción del aporte energético basado en azúcares, grasa y sodio. Según lo declarado por los productos convencionales, una bebida gaseosa contiene aproximadamente 107 kilocalorías, mientras que una bebida ofertada como light contiene de 0.5 a 3.5 kilocalorías (Sánchez, 2008). Una bebida light sustituye el azúcar por otro compuesto como los edulcorantes artificiales, entre ellos, la fructosa, el ciclamato, la sacarina y el aspartamo. Estos edulcorantes son de 100 a 200 veces más dulces que el azúcar blanco, se absorben a nivel intestinal y se excretan por vía renal sin metabolizarse. (L. Gómez, A. Jiménez, M. Bacardi, 2013)

Es decir, según investigaciones ralizadas sobre el aporte nutricional de los productos ofertados como light describen a estos alimentos como generadores a mediano y largo plazo de otro tipo de enfermedades crónicas no transmisibles como daño renal, hígado graso, la propensión a la diabetes y a problemas cardíacos y metabólicos serios, y advierten de su consumo ya que proporcionan grandes cantidades de calorías vacías y con poco beneficio nutricional. (Bray, 2012)

\section{Compra y consumo de bebidas light no alcohólicas}

Para Galán (2012) la relación compra - consumo es influenciada por factores que ayudan a la toma de decisiones para la adquisición de productos. Estos factores se consideran desde diferencias individuales como los segmentos de edad, niveles de educación, personalidad y actitudes, hasta características del entorno como niveles de demanda, perspectivas económicas, cambios tecnológicos, transformaciones políticas y regionales, desarrollo de la competitividad, entre otros. El análisis de estos factores permiten aclarar los procedimientos que utilizan las personas para tomar sus decisiones.

Los factores externos que inciden en la decisión y situación de compra son: el grupo demográfico al que el individuo pertenece, la prácticas culturales y grupos identitarios en los cuales las personas se desenvuelven, el nivel socio económico, los grupos de referencia como los medios de comunicación, masivos, alternativos, virtuales, entre otros; las mediaciones sociales y culturales como la familia, el entorno escolar, y por supuesto, las actividades de marketing y publicidad que las empresas utilizan para informar y persuadir al consumidor de realizar la compra de un producto específico. (Galán, 2012)

Todos estos factores externos están relacionados entre sí y con la sociedad de consumo; la ideología del estilo de vida light engloba los factores externos como consecuencia de las prácticas sociales y relaciones inter personales, en un mundo globalizado y cargado de necesidades subjetivas re creadas por la misma sociedad de consumo. 
Los factores internos: percepción, aprendizaje, memoria, motivaciones, emociones, personalidad y actitudes, influencian la decisión de compra y consumo de un sujeto inmerso en la sociedad del verse y sentirse bien, y están enmarcadas en el estilo de vida contemporáneo, en el cual el sentirse bien es un proceso subjetivo determinado por el entorno, también como un factor externo que interviene en las decisiones de consumo de la alimentación light.

A nivel mundial, el consumo de bebidas light no alcohólicas aumenta considerablemente en las sociedades globalizadas. Desde el año 2014 al año 2016, el 75\% de los cambios en el consumo de bebidas obedece a transformaciones en los estilos de vida del consumidor que promueve la adquisición de hábitos alimenticios que satisfagan estas necesidades dietéticas (Altonivel, 2014)

En el Ecuador existe un aumento en el consumo de productos ofertados en el mercado como bajos en calorías, light o ligeros. Desde el 2007 están ingresando al mercado nacional 88 variedades light en el segmento de alimentos y 38 alternativas en el rubro de bebidas, de tal forma que el $54.2 \%$ de los ecuatorianos han consumido en algún momento de su vida un producto o bebida light no alcohólica (Ecuador Inmediato, 2015).

Según las investigaciones realizadas por la empresa Ipsa, desde el año 2006 en el D.M de Quito ha crecido considerablemente la demanda por el consumo de bebidas artificiales (El Universo 2006), además existe un estudio concreto sobre el consumo de bebidas no alcohólicas principalmente las carbonatadas y jugos artificiales (Moreno, 2010), sin embargo no existe un estudio estadístico más específico sobre el consumo de bebidas light no alcohólicas, por lo que el campo de investigación se vuelve interesante ya que se puede evidenciar una gran variedad y oferta de este producto en tiendas de barrio, supermerados, restaurantes, discotecas, bares particulares y públicas de instituciones educativas, empresas, entre otros, lo que hace pensar que existe una importante demanda de bebidas light no alcohólicas, especialmente de la población joven quiteña.

Ante este contexto es preciso responder la interrogante ¿cómo se manifiestan las decisiones de compra y consumo de bebidas light no alcohólicas en el D.M. de Quito y cuál sería su relación con el estilo de vida light y la cultura del verse y sentirse bien? De lo que surge analizar el estilo de vida como factor externo que pueda incidir en las decisiones de compra y consumo de este producto, y finalmente determinar el nivel de información y conocimiento que los consumidores tienen sobre los beneficios o perjuicios de lo ofertado como alimentación light.

\section{Metodología}

El diseño de investigación corresponde a una metodología cuantitativa, no experimental, temporalidad transeccional y alcance descriptivo correlacional (R. Hernández, C. Fernández, M. Baptista, 2010). La técnica utilizada es la encuesta con el instrumento cuestionario estructurado, cuyas preguntas se enfocaron en la correlación de las variables: identificación y conocimiento del producto, causas de compra, cualidades ofertadas, frecuencia de compra y consumo, todo ello en relación a la edad y género de los encuestados.

\section{Población y muestra}

El universo investigado constituye la población económicamente activa del D. M. de Quito comprendidos entre las edades de 13 a 65 años de edad. Para la determinación del universo de estudio se establece la zona norte denominada Administración Zonal La Delicia. Según el Instituto de la Ciudad Quito (Raúl Moscoso, Fabián Regalado, 2010) esta zona es la de mayor extensión geográfica del Distrito Metropolitano de Quito, y la segunda en importancia en número de empresa y comercio que incluye: micro, pequeña, mediana y gran empresa, restaurantes, destinos turísticos, centros comerciales, entre otras. Además, esta zona es una alternativa de salida de la ciudad hacia otros destinos del país, incluyendo a uno 
de los mayores atractivos turísticos como la Mitad del Mundo. Los datos han sido tomados del Censo de Vivienda 2010 según registros del Instituto de Censos y estadísticas INEC (Censos, 2010).

Para determinar la muestra se utilizó la técnica del muestreo aleatorio no probabilístico con la fórmula para poblaciones finitas, dando como resultado 384 elementos muestrales. Los datos descriptivos de la muestra considerada se presentan en la tabla 1

\begin{tabular}{|c|c|c|c|c|c|}
\hline Población total & Fr & Porcentaje & Muestra & Fr & Porcentaje \\
\hline Hombres & 177.445 & $48 \%$ & Hombres & 184 & $48 \%$ \\
Mujeres & 191.004 & $52 \%$ & Mujeres & 200 & $52 \%$ \\
\hline Total & 368.449 & $100 \%$ & Total & $\mathbf{3 8 4}$ & $\mathbf{1 0 0 \%}$ \\
\hline
\end{tabular}

Tabla 1: Población y muestra

\section{Procedimiento}

El instrumento diseñado valoró 3 aspectos (a) las causas que intervienen en las decisiones de compra/consumo de las bebidas light no alcohólicas, (b) identificación y conocimiento del producto, (c) factores para la decisión de compra /consumo. Para su elaboración se procedió con los siguientes pasos: 1) Construcción de los ítems en relación al estado de arte que incluye la comprensión de la cultura del estilo de vida light como factor externo que interviene en las decisiones de compra / consumo en la sociedad contemporánea, además se tomó en cuenta la variable edad y género. 2) Validación del instrumento por pares profesionales expertos en el tema. 3) Aplicación de un estudio piloto con 10 personas que no fueron parte de la muestra. 4) Diseño final del instrumento.

La aplicación de la encuesta se realizó en el último trimestre del año 2016 en 3 centros comerciales del sector escogido para el estudio, por lo que se tuvo un contacto directo con la realidad del consumidor y su conducta de compra, así como su correspondencia con los diferentes tipos de productos que se encuentran en el mercado.

Cabe mencionar que en el momento de la aplicación de la encuesta no fue posible aplicar a la muestra exacta escogida, sino por la predisposición que el género femenino y masculino tuvieron en el momento de presentar el instrumento. Así, el género femenino tuvo mayor predisposición para contestar el cuestionario. En la tabla 2 se presenta la frecuencia y porcentaje de participación por cada género.

\begin{tabular}{|c|c|c|}
\hline Género & Frecuencia (fr) & Porcentaje (\%) \\
\hline Femenino & 225 & 51,84 \\
Masculino & 159 & 48,16 \\
Otro & 0 & 0 \\
\hline Total & 384 & 100 \\
\hline
\end{tabular}

Tabla 2: Participación en la encuesta del género femenino y género masculino

\section{Resultados}

El grupo poblacional que tiene mayor preferencia por el consumo de bebidas light no alcohólicas se encuentra entre los 24 y 29 años, las mujeres de este grupo son quienes más consumen este producto con el $24,3 \%$, frente al $22,5 \%$ de consumidores hombres. Es interesante observar que de todas las edades es el género femenino quien más consume las bebidas light no alcohólicas con el 51,9\% frente al 48,1\% de consumo por parte del género masculino. Se pueden apreciar los datos en la tabla 3. 


\begin{tabular}{|c|c|c|c|}
\hline $\begin{array}{c}\text { Preferencia de } \\
\text { consumo por edad }\end{array}$ & $\begin{array}{c}\% \\
\text { Muestra } \\
\text { total }\end{array}$ & $\begin{array}{c}\% \\
\text { Femenino }\end{array}$ & $\begin{array}{c}\% \\
\text { Masculino }\end{array}$ \\
\hline 13 a 17 años & 2,3 & 1,2 & 1,1 \\
\hline 18 a 23 años & 13,1 & 6,8 & 6,3 \\
\hline 24 a 29 años & 46,8 & 24,3 & 22,5 \\
\hline 30 a 35 años & 25,2 & 13,1 & 12,1 \\
\hline 36 a 49 años & 10,2 & 5,3 & 4,9 \\
\hline Mayores de 50 años & 2,3 & 1,2 & 1,1 \\
\hline Total & 100,0 & 51,9 & 48,1 \\
\hline
\end{tabular}

Tabla 3. Porcentaje de preferencia de consumo de bebidas light no alcohólicas según la variable edad y género.

Respecto a la frecuencia de consumo, se evidencia que de la muestra total encuestada, el 88,02\% consume con frecuencia las bebidas light no alcohólicas, esto es al menos 3 veces a la semana. De esta cifra, el 58.9\% son del género femenino que sí consume frecuentemente este producto, versus el 54,64\% del género masculino. En la tabla 4 se puede revisar los datos obtenidos.

\begin{tabular}{|c|c|c|c|}
\hline $\begin{array}{l}\text { Consumo frecuente } \\
\text { (al menos } 3 \text { veces a la } \\
\text { semana) de bebidas } \\
\text { light no alcohólicas }\end{array}$ & $\%$ Muestra total & $\begin{array}{c}\% \\
\text { Femenino }\end{array}$ & $\%$ Masculino \\
\hline $\mathrm{Si}$ & 88,02 & 58,9 & 54,64 \\
\hline No & 11.98 & 41,1 & 45,36 \\
\hline Total & 100 & 100 & 100 \\
\hline
\end{tabular}

Tabla 4. Frecuencia de consumo de bebidas light no alcohólicas con la variable Género En cuanto al nivel de identificación del producto por el envase o etiqueta, los resultados arrojaron que el $83,33 \%$ de encuestados creen ubicar el producto a la hora de decidir su compra y consumo, los datos se observan en la tabla 5 .

\begin{tabular}{|c|c|}
\hline $\begin{array}{c}\text { Identificación de bebidas light no } \\
\text { alcohólicas en el mercado por el envase o } \\
\text { su etiqueta }\end{array}$ & \% Muestra total \\
\hline $\mathrm{Si}$ & 83,33 \\
$\mathrm{No}$ & 16,67 \\
\hline Total & 100 \\
\hline
\end{tabular}

Tabla 5. Identificación del producto bebidas light no alcohólicas 
Sin embargo, el $66.67 \%$ de los encuestados consideran que una bebida light no alcohólica está implícita en aguas naturales y minerales, bebidas energizantes, aguas saborizadas, tés y jugos artificiales. De este grupo, el 34,60\% corresponde al género femenino, y el 32,07\% al género masculino. Así, solo el 33.33\% de la muestra total conoce las propiedades de las bebidas light no alcohólicas. Se observa el resultado en la tabla 6

\begin{tabular}{|c|c|c|c|}
\hline $\begin{array}{c}\text { Conocimiento de bebidas light no } \\
\text { alcohólicas }\end{array}$ & $\begin{array}{c}\text { \% } \\
\text { Muestra total }\end{array}$ & \% Femenino & $\begin{array}{c}\text { \% } \\
\text { Masculino }\end{array}$ \\
\hline $\begin{array}{c}\text { Aguas naturales y minerales, bebidas } \\
\text { energizantes, jugos artificiales, aguas } \\
\text { saborizadas y tés }\end{array}$ & 66,67 & 34,60 & 32,07 \\
$\begin{array}{c}\text { Bebidas sometidas a procesos de reducción } \\
\text { del aporte energético (azúcares) }\end{array}$ & 33,33 & 17,30 & 16,03 \\
\hline \multicolumn{2}{|c|}{ Total } & $\mathbf{1 0 0}$ & $\mathbf{1 0 0}$ \\
\hline
\end{tabular}

Tabla 6. Conocimiento del producto bebidas light no alcohólicas con variable género

Sobre las causas de compra y consumo de bebidas light no alcohólicas, el factor salud es la principal motivación con el 80,99\% del total de la muestra, de los cuales, el 42,03\% corresponde al género femenino, mientras que los factores hidratación y disminución del valor energético no son considerados como causas de motivación para la compra y consumo de estos productos. En cuanto a la cualidad como causa, el factor hidratación tiene el 77,34\% de preferencia de la muestra total, de los cuales el 40,14\% es del género femenino, mientras que el factor disminución del valor energético es considerado como una cualidad de causa por el $22,66 \%$ de la muestra total, siendo el 11,66\% de género femenino. En la tabla 7 se observan estos resultados.

\begin{tabular}{|c|c|c|c|c|}
\hline $\begin{array}{c}\text { Causas de } \\
\text { compra/consumo de } \\
\text { bebidas light no } \\
\text { alcohólicas }\end{array}$ & Factor & $\begin{array}{c}\% \\
\text { Muestra total }\end{array}$ & $\begin{array}{c}\% \\
\text { Femenino }\end{array}$ & $\%$ Masculino \\
\hline \multirow{3}{*}{ Motivación } & Salud & 80,99 & 42,03 & 38,96 \\
\hline & Hidratación & ---- & ---- & --- \\
\hline & $\begin{array}{l}\text { Disminución del } \\
\text { valor energético }\end{array}$ & ---- & ---- & ---- \\
\hline \multirow{3}{*}{ Cualidad } & Salud & ---- & ---- & ---- \\
\hline & Hidratación & 77,34 & 40,14 & 37,2 \\
\hline & $\begin{array}{l}\text { Disminución del } \\
\text { valor energético }\end{array}$ & 22,66 & 11,76 & 10,9 \\
\hline
\end{tabular}

Tabla 7. Causas de compra / consumo de bebidas light no alcohólicas y factores que inciden en el consumo por género. 
Paralelamente a este resultado, el 92,1\% de encuestados del género masculino consideran que el consumo frecuente de bebidas light no alcohólicas mejorará su bienestar físico y corporal, mientras que el 93,64\% del género femenino piensan que el consumo frecuente de bebidas light no alcohólicas mejorará la imagen física a largo plazo, versus el $62.79 \%$ del género masculino que considera que esto no sucederá. Se puede observar este resultado en la tabla 8.

\begin{tabular}{|l|c|c|c|c|}
\hline \multirow{2}{*}{ Ítem } & \multicolumn{2}{|c|}{ \% Femenino } & \multicolumn{2}{c|}{ \% Masculino } \\
\cline { 2 - 5 } & $\mathrm{Si}$ & No & $\mathrm{Si}$ & No \\
\hline
\end{tabular}

El consumo frecuente de bebidas light no alcohólicas mejorará el bienestar físico y corporal
83,43
16,56
92,10
7,90

El consumo frecuente de bebidas light no alcohólicas mejorará la imagen $\begin{array}{llll}93,64 & 6,36 & 37,21 & 62,79\end{array}$

Tabla 8. Bienestar físico, corporal e imagen física con variable género

Finalmente el $62.8 \%$ de consumidores han decidido la compra y consumo de bebidas light no alcohólicas gracias a lo publicitado respecto a la disminución calórica de la bebida original, y el 74.7\% desconoce los perjuicios de los componentes sustitutos del azúcar (sacarina, fructosa, aspartame) introducidos en la bebida light, tanto en género masculino como femenino.

\section{Discusión y Conclusiones}

A nivel mundial las actividades de marketing y publicidad de la industria alimenticia utiliza estrategias y mecanismos de información y persuasión hacia los consumidores, con el fin de determinar las cualidades y características de los productos a los que se les atribuye significados sociales y simbólicos, entre ellos los productos considerados "saludables" como las bebidas light (Rico, 2011). Esta percepción, asumida por el consumidor, es un factor interno que incide en la decisión de compra y consumo de las bebidas light no alcohólicas, ya que se introduce el beneficio ofertado, es decir, la obtención de la salud a partir de la hidratación, con el beneficio adicional de consumir menos calorías. En este sentido, el consumidor no tiene que hacer ningún sacrificio para mantenerse "saludable" ya que su bebida favorita, en versión light, le proporciona buen sabor y placer sin calorías.

El consumo de estos productos ofertados como light también entran en el esquema de lo simbólico, significa ser parte de un grupo de referencia, significa estar conectado con el estilo de vida light que le 
llevará a la salud y al bienestar físico. Se trata de una integración social que encierra intereses y hábitos particulares (Bourdieu, 1988)

Pues bien, en respuesta a la ideología del estilo de vida, la industria de productos alimenticios light en el D. M. de Quito responde a la creciente demanda en el mercado de consumo, principalmente de bebidas light no alcohólicas. Este consumo consciente se puede leer como la preocupación por aplacar, detener o prevenir las denominadas enfermedades del siglo XXl, las relacionadas con la mala calidad de vida como consecuencia del exceso de trabajo, el estrés, la alimentación procesada, el sedentarismo y las dinámicas propias de la vida moderna urbana. Galán (2012) en su investigación sobre cultura light y estilo de vida obtuvo resultados que evidenciaron la necesidad, casi obsesiva, de las personas por compensar estas dinámicas llevando una vida light a través de diferentes prácticas como el deporte y principalmente con hábitos alimenticios que estén acordes al estilo de vida light, y esto es principalmente en las poblaciones jóvenes.

En el presente estudio el grupo poblacional con mayor hábito de compra y consumo de bebidas light no alcohólicas es la comprendida entre los 24 y 35 años de edad, se entiende así que la población joven, económicamente activa y con actividades laborales sería la más preocupada por mantenerse en "forma" y estar físicamente saludables, pero además existe la preocupación por mantener una buena imagen, es decir, un cuerpo acorde a las exigencias de la sociedad que venera la "buena" estética. En este sentido habría que analizar lo que Arízaga (2004) debate en su investigación sobre el interés por mantener la salud y el bienestar físico para competir y "estar" aptos física y estéticamente en el mundo laboral y en la vida pública.

Por otra parte, la investigación realizada revela datos muy interesantes sobre la identificación y el conocimiento del producto, es decir, considerando que existe un consumo frecuente de bebidas light no alcohólicas y que la mayoría de encuestados tanto del género masculino como del femenino, creen identificar estos productos en el mercado por los envases y las etiquetas expuestas, existe un gran porcentaje de consumidores que desconoce lo que realmente es una bebida light, desconoce los procesos químicos a los cuales son sometidos, y desconoce los perjuicios que podrían ocasionar su consumo frecuente. Para la gran mayoría de encuestados una bebida energizante, un jugo artificial o simplemente el agua puede ser considerada light.

Esto implica de alguna manera que lo natural puede ser calificado light, o que se ignore los procesos de compensación de azúcar por edulcorantes artificiales. P. Bourdieu en su teoría sociológica sobre el habitus manifiesta que las personas tienen una estrecha relación con su entorno social, y que este es dinamizado a través de prácticas estructuradas y estructurantes mediadas por la institucionalidad operante y dominante (C. Dendasck, G. Lee, 2016). En este caso la estructura es toda la industria dedicada a la comercialización y promoción de estos productos. Significa que el consumidor no necesariamente toma la decisión de comprar y consumir una bebida light no alcohólica por sus valores o beneficios alimenticios, sino por la etiqueta en el envase que es promocionada como "saludable", lo que le certifica mantener el sabor original pero con salud. La estructura opera ofreciéndole al consumidor todos los beneficios, salud, hidratación, bajas calorías, y en consecuencia bien - estar físico, estético y emocional, lo propio de la cultura y estilo de vida light.

Es importante recalcar que para el género femenino, la salud, la estética y la hidratación que promueven el consumo de estos productos se ha convertido en un hábito necesario, así lo revela la presente investigación y se compara con un estudio realizado por el grupo Protegeles (2009) y Palacio (2009), cuyos resultados no difieren en gran medido de los obtenidos en la presente investigación y se demuestra que más del $80 \%$ de las mujeres consumen productos light para mantener una figura esbelta, creyendo que alcanzarán la felicidad como lo muestra la publicidad (Rico, 2011). 
También es preciso recalcar que para el género masculino no es la imagen física y/o estética lo que motiva a la decisión de compra y consumo de bebidas light no alcohólicas, mas bien el bienestar físico y corporal, característica que también es publicitada en el mercadeo del producto, e incidiría en el imaginario de aptitud física para el mundo laboral (Arízaga, 2004).

Finalmente es preciso concluir que las decisiones de compra y consumo de bebidas light no alcohólicas en el D. M. de Quito, tomando como referencia la zona La Delicia, se manifiestan en concordancia con el estilo de vida light, primero por la necesidad de adquirir un cuerpo apto para el mundo moderno en la dualidad verse/sentirse bien, es decir, vivir saludable y socialmente apto para el mundo laboral, luego, acorde a las exigencias físicas en el caso del género masculino y estéticas en el caso del imaginario femenino. La industria actual recoge estas manifestaciones socio - culturales y las transforma en necesidades materializadas en productos que las satisfagan. Sin embargo, existe un desconocimiento generalizado sobre las características de estos productos, pues la industria implicada trabaja con las subjetividades, como dice Ravettino (2017)

El estilo de vida posee una dimensión subjetiva en tanto forma de percepción y aspiración, no obstante su alcance no se limita al campo de la subjetividad sino que está profundamente vinculado con la estructura de la vida social, política y económica constituyendo una gran red de industrias implicadas. (Ravettino, 2017)

Pese a que la presente investigación no revela datos específicos sobre características socio demográficas como ocupación, nivel de estudio, profesión, entre otras, parece indispensable realizar una investigación complementaria que evidencie precisamente la actitud de elección y consumo de bebidas light no alcohólicas según estas características.

\section{Referencias bibliográficas}

Altonivel. (2014). Productos Light, un nicho con potencial. Obtenido de http://www.altonivel.com.mx/productos-light-un-nicho-con-potencial.html Consultado el $3 / 11 / 2014$

Arízaga, C. (2003). Ciudad y usos del espacio en los jóvenes: el consumo juvenil desde dos escenarios urbanos. En Wortman, Pensar en las clases medias. Consumos culturales y estilos de vida urbanos en la Argentina de los noventa. Buenos Aires: La Crujía.

Arízaga, C. (2004). Cambios profundos en los modelos sociales del mundo laboral. La nueva ética del trabajo. Debate, 38 - 40.

Bauman, Z. (2003). Trabajo, consumismo y nuevos poderes. Barcelona: Gedisa.

Boltvinik, J. (s.f.). Max Neef: una teoría de las necesidades humanas en desarrollo. Obtenido de http://www.julioboltvinik.org/documento/tesis\%20Ampliar

Bourdieu, P. (s.f.). Espacio social y poder simbólico. En P. Bourdieu, Cosas dichas. Buenos Aires: Gedisa.

Bray, G. (2012). Fructose and Risk of Cardiometabolic Disease. Current Atherosclerosis Reports, 570 578.

Carla Dendasck, Gilead Lee. (2016). Concepto de Habitus de Pierre Bourdieu y Norbert Elías. Revista científica multidisiplinaria base de conocimiento, 1 - 10.

Censos, I. N. (2010). El nuevo INEC. Obtenido de http://www.inec.gob.ec/nuevo_inec/cpv.html

Galán, S. (2012). Estudio del comportamiento del consumidor de entre 20 y 25 años en la adquisición de productos de baja impolicación para el 2012. Quito: PUCE. 
Inmediato, E. (2015). Oferta light aumenta en Ecuador. Obtenido de http://ecuadorinmediato.com/index.php?module=Noticias\&func=news_user_view\&id=55749\&u mt=expreso_guayaquil_oferta_light_aumenta_en_ecuador Consultado el 20/07/2015

L. Gómez, A. Jiménez, M. Bacardi. (2013). Estudios aleatorizados sobre el efecto del consumo de bebidas azucaradas sobre la adiposidad en adolescentes. Nutrición Hospitalaria, 1792 - 1796.

Moreno, A. (2010). Desarrollo de canales de mercadeo mediante la identificación de las ocasiones de compra/consumo de bebidas no alcohólias de los quiteños. Quito: ESPOL.

Raúl Moscoso, Fabián Regalado. (2010). Conociendo Quito. Estadísticas del Distrito Metropoltano. Quito: La Caracola Editores.

Ravettino, D. R. (2017). Cultura light: estilo de vida y represntaciones sociales en los sectores medios urbanos. Buenos Aires: Digital.

Rico, J. (2011). La influencia de la publicidad en la toma de decisión de compra en los productos light en el consumidor. Observatorio Calasanz, 261 - 270.

Roberto Hernández, Carlos Fernández, María Baptista. (2010). Metodología de la Investigación. México D.F.: Mc Graw Hill.

Sánchez, A. (2008). Productos que se dicen light. Consumidor, 39 - 59.

Universo, E. (s.f.). Los gustos marchan de formas distintas en las urbes. Obtenido de http://www.eluniverso.com/2008/01/06/0001/9/5275DB3308EC4732A06399983E3AF332 Consutlado el 26/11/2014

V. Malik, B. Popkin, G. Bray, J. Després, F. Hu. (2010). Sugar Sweetened Beverages, Obesity, Tipe 2 Diabetes and Cardiovascular Disease risk. Circulation, 1356 - 1364. 